\title{
Frequency Domain Acquisition Method for Dual-System GNSS
}

\author{
Zhang Zhan, Xiong Zhang, Zhang Xiaolin, Su Linlin \\ School of Electronic and Information Engineering \\ Beihang University \\ Beijing, China \\ Email: Johnnyz@vip.163.com
}

\begin{abstract}
In Global Navigation Satellite System (GNSS), acquisition process adjusts the code phases of the incoming signal and the local pseudo-random sequence of the corresponding satellite to less than a code chip offset, and generate the correct local carrier frequency. In BD2/GPS Dual-System GNSS, the code chip lengths are different between two systems and the satellite channels are doubled. By using FFT, a fast acquisition method is presented. It is shown that the method can be used for multi-channel and multilength satellite signals acquisition at one time, the speed is significantly improved and the complexity is reduced than time domain acquisition method.
\end{abstract}

Keywords-GNSS; Acquisition; Dual-System; FFT

\section{INTRODUCTION}

$\mathrm{BD} 2$ is the 2nd generation of Chinese satellite navigation system. By the year of 2020, there will be more than 30 satellites serve this system, but before that the BD2/GPS Dual-System GNSS is needed to fill the gap.

In the space, as the noise completely masks the GNSS signals, the receivers use a method called correlation to process against the noise. The correlation synchronizes the local signals in the receiver with the signals received in order to identify the transmitting satellites and propagation delay. To achieve code synchronization, it usually takes two steps: acquisition and tracking.

The purpose of acquisition is to determine how much propagation delay and how much the Doppler frequency is. The receiver should search a large range to find the most matched parameter to set local oscillator.

A conventional way is to use a time domain correlate [3], which can multiply the input signal with local signal chip by chip and sum to get a cumulative correlation result, once the result exceeds a threshold, the right parameter is found. Obviously it needs a long calculation buffer and time. In dual-system GNSS, different chip length needs different corrector, and every signal channel also needs a single corrector. We found it occupied large FPGA resources and caused large group delay.

Since complexity in the number of arithmetic operations can be reduced algorithmically by removing redundant calculations [1], we found DFT can be used to compute the correlation for all the code phases and channels at once at a candidate frequency. Alternatively, DFT can be used for the parallel search in frequency dimension at a candidate code chip phase.

\section{CONVENTIONAL ACQUisition METHOD}

The conventional time domain acquisition method is shown in figure. 1

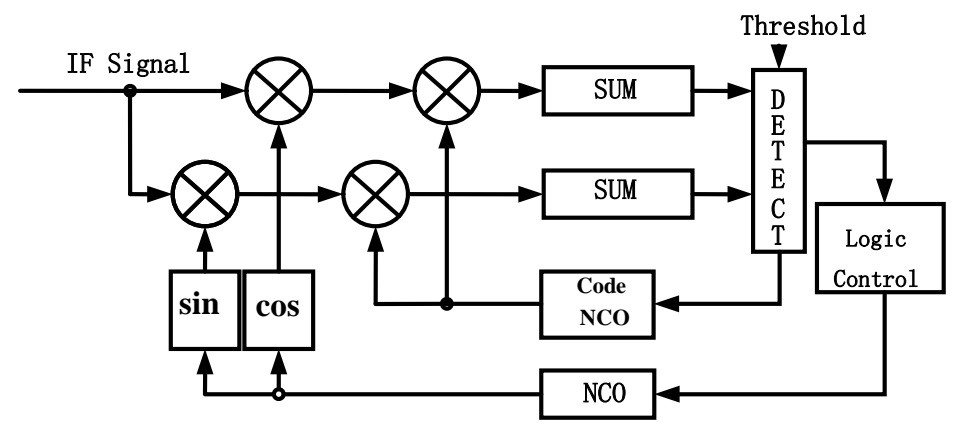

Figure 1. Conventional time domain acquisition

The IF (intermediate-frequency) signal received from the satellites after $\mathrm{A} / \mathrm{D}$ sampling can be presented by

$$
s(n)=A D\left(n t_{s}-\tau_{n}\right) C\left(n t_{s}-\tau_{n}\right) \cos \left[2 \pi\left(f_{I F}+f_{d}\right)\left(n t_{s}-\tau_{n}\right)+\phi_{0}\right]+N^{\prime}\left(n t_{s}\right)
$$

$A$ is the amplitude, $D(t)$ is data sequence, $C(t)$ is code chip, $t_{s}$ is sample period, $\tau_{\mathrm{n}}$ is chip delay, $\mathrm{f}_{\mathrm{IF}}$ is IF frequency, $f_{d}$ is Doppler frequency, $\varphi_{0}$ is carrier initial phase, $N^{\prime}$ is noise.

Assume $\hat{\tau}_{0}, \hat{f}_{d}, \hat{\phi}_{0}$ are the estimated chip phase, Doppler frequency and carrier initial frequency, then the local I, Q signals are

$$
\begin{aligned}
& s_{I}(n)=C\left(n t_{s}-\hat{\tau}_{0}\right) \cos \left[2 \pi\left(f_{I F}+\hat{f}_{d}\right)\left(n t_{s}-\hat{\tau}_{0}\right)+\hat{\phi}_{0}\right] \\
& s_{Q}(n)=C\left(n t_{s}-\hat{\tau}_{0}\right) \sin \left[2 \pi\left(f_{I F}+\hat{f}_{d}\right)\left(n t_{s}-\hat{\tau}_{0}\right)+\hat{\phi}_{0}\right]
\end{aligned}
$$

The (n)th correlated I,Q signals are

$$
\begin{aligned}
& I(n)=\sum_{n=0}^{N-1} s(n) s_{I}(n)=\frac{A D(n) T}{2 t_{s}} R\left(\Delta \tau_{n}\right) \operatorname{Sa}\left(\pi \Delta f_{n} T\right) \cos \left(\Delta \phi_{n}\right) \\
& Q(n)=\sum_{n=0}^{N-1} s(n) s_{Q}(n)=\frac{A D(n) T}{2 t_{s}} R\left(\Delta \tau_{n}\right) \operatorname{Sa}\left(\pi \Delta f_{n} T\right) \sin \left(\Delta \phi_{n}\right)
\end{aligned}
$$


$\mathrm{Sa}(\mathrm{x})=\sin (\mathrm{x}) / \mathrm{x}, \mathrm{T}$ is cumulative time, $\Delta \tau_{\mathrm{n}}$ is difference time of local and input signal, $\Delta \mathrm{f}_{\mathrm{n}}$ is difference frequency of local and input signal, $\varphi_{0}$ is difference of chip phase.

The auto correlative function of GNSS pseudo-random sequence is

$$
R(\tau) \approx \begin{cases}1-\frac{|\tau|}{T_{c}}, & |\tau| \leq T_{c} \\ 0, & |\tau|>T_{c}\end{cases}
$$

Let $V_{j}=I_{j}^{2}+Q_{j}^{2}$, ignore the noise, then

$$
\begin{gathered}
V_{j}=I_{j}{ }^{2}+Q_{j}{ }^{2}=\frac{A^{2} T^{2}}{4 t_{s}^{2}} R(\Delta \tau)^{2} \sin \mathrm{c}^{2}(\pi \Delta f T) \\
V=\sum_{j} V_{j}
\end{gathered}
$$

$V$ is the cumulative sum of square of I,Q signal. Compare it with the threshold, if exceeds the threshold, the search is done; otherwise reset the NCO (numerically controlled oscillator) parameter and do the correlation again.

\section{FREQUENCY DOMAIN ACQUISITION}

\section{A. Theory}

According to the signal processing theory, the time domain correlation operation is equivalent to the frequency domain multiplying, so in the acquisition module, the input data is processed by FFT and multiply to approach the equivalent correlation result.

$\mathrm{N}$ points cumulative correlation operation can be presented by:

$$
\begin{aligned}
& I(n)=\sum_{n=0}^{N-1} s(n) C\left(t_{n}-\hat{\tau}\right) \cos \left(2 \pi\left(f_{I F}+\hat{f}_{d}\right) t_{n}\right) \\
& Q(n)=\sum_{n=0}^{N-1} s(n) C\left(t_{n}-\hat{\tau}\right) \sin \left(2 \pi\left(f_{I F}+\hat{f}_{d}\right) t_{n}\right)
\end{aligned}
$$

$t_{n}=t_{0}+n T_{s}, t_{0}$ is the sample start time, $T_{s}$ is sample period.

In the acquisition process, if at $\left(\hat{t}_{s}, \hat{f}_{d}\right)$ the threshold of $V\left(\hat{t}_{s}, \hat{f}_{d}\right)=I^{2}+Q^{2}$ is found, the searching process is end, otherwise the signal is lost.

Assume it is a uniform sampling process, as $T_{s}=t_{n+1}-t_{n}$

Then there has $\begin{gathered}t_{n}=t_{0}+n \cdot T_{s} \\ \hat{\omega}=2 \pi \hat{f}_{d}\end{gathered}, \hat{\tau}=t_{0}+m \cdot T_{s}, \hat{\omega}=2 \pi \hat{f}_{d}$

Define

$$
\begin{aligned}
& z\left(n, \hat{w}_{d}\right)=z\left(t_{0}+n \cdot T_{s}, \hat{w}_{d}\right)=I+j Q \\
& =\sum_{n=0}^{N-1} s(n) C\left[n T_{s}-m T_{s}\right]\left\{\cos \left[\left(\omega_{I}+\hat{\omega}_{d}\right) t_{n}\right]-j \sin \left[\left(\omega_{I}+\hat{\omega}_{d}\right) t_{n}\right]\right\} \\
& =\sum_{k=0}^{N-1} s(n) c_{n-m} \exp \left[-j\left(\omega_{I}+\hat{\omega}_{d}\right) t_{n}\right]
\end{aligned}
$$

Let $y(n)=s(n) \exp \left[-j\left(\omega_{I}+\hat{\omega}_{d}\right) t_{n}\right]$, then $z\left(n, \hat{w}_{d}\right)$ is the circular correlation result of $y(n)$ and $c_{n-m}$, the time domain circular correlation can be transformed to frequency domain:

$r_{x y}(m)=\operatorname{IFFT}\left(R_{x y}(K)\right)=\sum_{k=0}^{N-1} x(k) y^{*}((k-m))_{N} \Leftrightarrow R_{x y}(K)=X(K) Y^{*}(K)$

$X(K), Y(K)$ is the FFT result of $x(k), y(k)$.

Then it is able to calculate $z\left(n, \hat{w}_{d}\right)$ in frequency domain

$$
\begin{gathered}
{\left[\begin{array}{c}
S_{0} \\
S_{1} \\
\vdots \\
S_{N-1}
\end{array}\right]=F F T\left(\left[\begin{array}{c}
s_{0} \exp \left[-j\left(w_{I}+\hat{w}_{d}\right) t_{0}\right. \\
s_{1} \exp \left[-j\left(w_{I}+\hat{w}_{d}\right) t_{1}\right. \\
\vdots \\
s_{N-1} \exp \left[-j\left(w_{I}+\hat{w}_{d}\right) t_{N-1}\right.
\end{array}\right]\right)} \\
{\left[\begin{array}{c}
C_{0} \\
C_{1} \\
\vdots \\
C_{N-1}
\end{array}\right]=F F T\left(\left[\begin{array}{c}
c_{0} \\
c_{1} \\
\vdots \\
c_{N-1}
\end{array}\right]\right)}
\end{gathered}
$$

$z\left(n, \hat{w}_{d}\right)(\mathrm{n}=0,1, \cdots, \mathrm{N}-1)$ can be calculated by

$$
\left[\begin{array}{c}
z\left(0, \hat{w}_{d}\right) \\
z\left(1, \hat{w}_{d}\right) \\
\vdots \\
z\left(N-1, \hat{w}_{d}\right)
\end{array}\right]=\operatorname{IFFT}\left(\left[\begin{array}{c}
C_{0}^{*} S_{0} \\
C_{1}^{*} S_{1} \\
\vdots \\
C_{N-1}^{*} S_{N-1}
\end{array}\right]\right)=\operatorname{IFFT}\left(\left[\begin{array}{c}
C_{0} S_{0} \\
C_{N-1} S_{1} \\
\vdots \\
C_{1} S_{N-1}
\end{array}\right]\right.
$$

The amplitude of $z\left(n, \hat{w}_{d}\right)$ is

$$
V\left(n, \hat{w}_{d}\right)=I^{2}(n)+Q^{2}(n)=\left|z\left(n, \hat{w}_{d}\right)\right|^{2} \quad \mathrm{n}=0,1, \cdots, \mathrm{N}-1
$$

If $V\left(n, \hat{w}_{d}\right)$ exceeds the threshold, then $\left(n, \hat{w}_{d}\right)$ is the estimated chip phase shift and Doppler frequency.

\section{B. Implementation of the Acquisition Module}

The work flow of frequency domain acquisition is shown in figure. 2

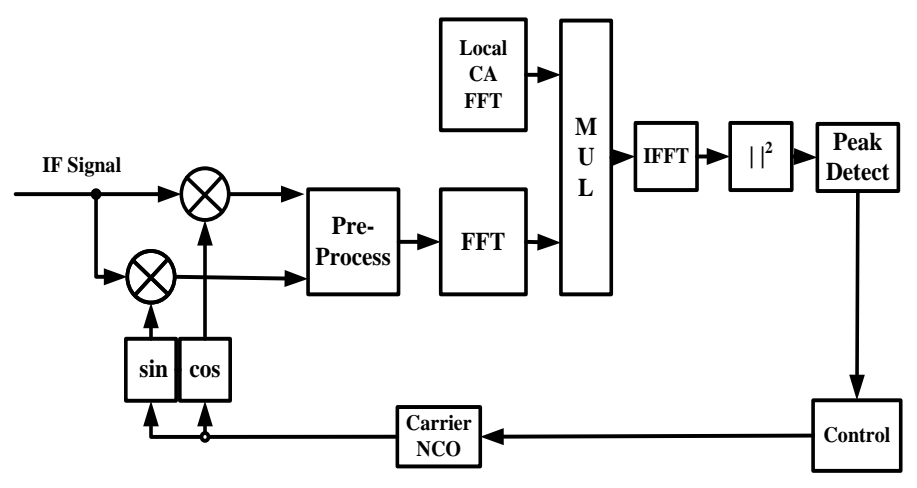

Figure 2. Work flow of frequency domain acquisition 
Acquisition module uses pipeline work, so the FFT and IFFT operations are carried out separately, the FFT and IFFT module are no need to work at the same time, this design decrease the work load of FPGA. The local CA FFT results are already saved to local ROM.

The frequency is controlled by NCO. At the start, the IF frequency is set to NCO control word, mix the generated local IF carrier with the input IF signal, then we get I, Q down conversion signal. The I, Q signal needs to be sampled $2 \mathrm{k}$ or $4 \mathrm{k}$ times and pre-process to fit the 2-based FFT requirements.

After pre-processed, the signal is transformed to frequency domain and multiplies by the local frequency domain CA chips. The result is then compared to the correlation peak threshold. If exceeds, the searching is done; otherwise increase or decrease the NCO frequency by $500 \mathrm{~Hz}$ and repeat searching. Usually the Doppler frequency is $\pm 10 \mathrm{kHz}$, so normally the searching ends after 40 repeats, and the NCO turns into tracking mode.

The computing procedure of frequency domain acquisition is:

(1) According to (10), transform $\mathrm{C}(\mathrm{n})$ to frequency domain $C_{m}=F F T[C(n)]$;

(2) Define a start searching frequency $\hat{w}_{d}$ by giving NCO control word. Get $\mathrm{N}$ point samples of input signal in one chip period and calculate the frequency spectrum by $S_{m}=F F T[y(n)], m=0,1, \cdots, N-1$;

(3) According to (11), calculate $z\left(n, \hat{w}_{d}\right), n=0,1, \cdots, N-1$;

(4) According to (12), calculate $V\left(n, \hat{w}_{d}\right), n=0,1, \cdots, N-1$

(5) Compare $V\left(n, \hat{w}_{d}\right) n=0,1, \cdots, N-1$ with threshold, if exceeds, then end the search; otherwise, increase $\hat{w}_{d}$ by step of $500 \mathrm{~Hz}$, repeat (2)-(5) ;

(6) Find the $\left(n, \hat{w}_{d}\right)$ that makes maximum $V\left(n, \hat{w}_{d}\right)$, then this $n$ is the chip phase shift, and the $\hat{w}_{d}$ is the Doppler frequency. Set the NCO to correct number to finish acquisition process.

\section{RESULTS}

We use Matlab to simulate the FFT acquisition module. The input signal is GPS L1 signal, receiver sample frequency is $5 \mathrm{MHz}$, so there are 5000 samples for each CA chip period. The IF frequency is $1.25 \mathrm{MHz}$, set phase delay to 2006 chips, Doppler frequency to $3208 \mathrm{~Hz}$, the SNR of IF signal to $-15 \mathrm{~dB}$. The simulation results are shown below, the chip phase shift and Doppler frequency is correctly calculated.

For one frequency point, it needs $\frac{N}{2}\left(\log _{2} N\right)$ times of complex multiplication to calculate $z\left(n, \hat{w}_{d}\right)$ and $S_{m}$, needs
$\mathrm{N}$ times of complex multiplication to calculate $S_{m} C_{m}$. So the total computation of FFT, IFFT is $N+N\left(\log _{2} N\right)$.

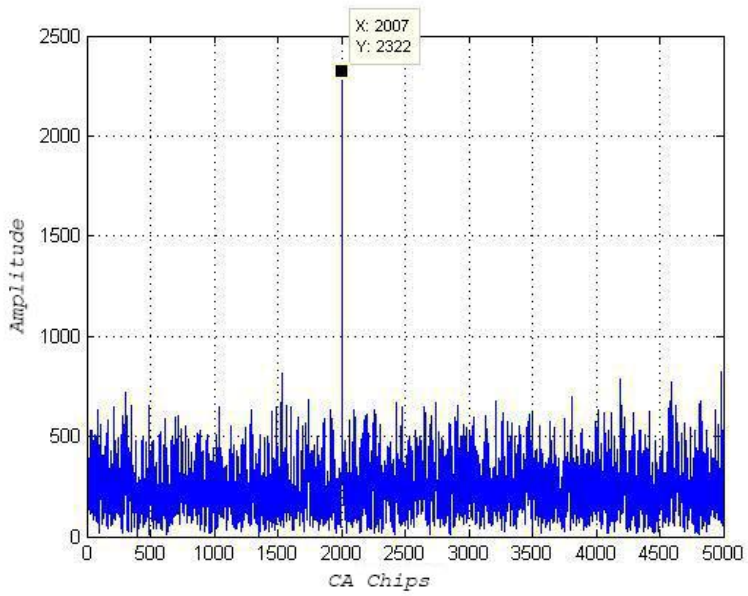

Figure 3. Correlation peak of frequency domain acquisition

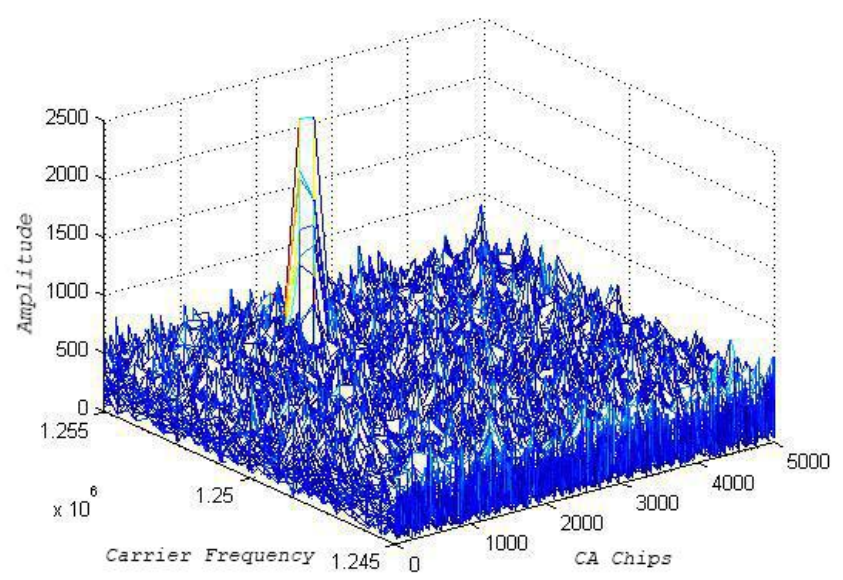

Figure 4. Correlation peak of two dimension search

If the searching step is $500 \mathrm{~Hz}$, then it needs 12 frequency points to cover the Doppler frequency range, such as $12\left[N+N\left(\log _{2} N\right)\right]$ times of to complex multiplication calculate $z\left(n, \hat{w}_{d}\right)$. Assume there are $N=4 k$ ( $k$ is FFT and IFFT points) samples in $1 \mathrm{~ms}$, then total computation is $52 \mathrm{k}$ times complex multiplication, if we use correlative accumulation method in time domain, the computation would be $(4 k)^{2}=16 k^{2}$, if $k=1024$ points, then the frequency domain method is 315 times faster than time domain method.

\section{CONCLUSION}

Since BD2/GPS Dual-system GNSS is combined by two navigation systems, it is more complex than conventional GNSS, if use conventional time domain acquisition process methods, the system would be redundant and ineffective. This paper analyzed the theories and algorithms of time and 
frequency domain acquisition methods, then presented a fast frequency domain based method, which significantly reduced the calculation complexity. Simulation shows it quicken the acquisition system speed to 315 times when using 1024 FFT.

Furthermore, since the FFT modules usually occupy big FPGA resources, we use reconfigurable FFT module in the system[5] to further improve the performance and reduce the cost.

\section{REFERENCES}

[1] Soltanian, B. The effect of the incoming signal decimation on the performance of the FFT-based acquisition stage in SDR GNSS receivers[A]. 5th ESA Workshop on Satellite Navigation Technologies and European Workshop on GNSS Signals and Signal Processing (NAVITEC)[C]. 2010:1-5
[2] Li Yang. Combining FFT and Circular Convolution Method for High Dynamic GPS Signal Acquisition[A]. The Eighth International Conference on Electronic Measurement and Instruments[C]. 2007:2159:1-162.

[3] Huang Lei. Study on GNSS Receiver Design and Signal Acquisition Technology[D]. Beihang University Press, Beijing, China

[4] Zhang Xiaolin, Zhang Zhan, Su Linlin, Zhang Shuai. BD/GPS DualSystem GNSS Receiver Baseband ASIC Chip BHBDGPS1002. Chinese Patent 08500489.8 Release date: Oct.13 2008.

[5] Su Linlin, Zhang Xiaolin, FFT Reconfigurable design in Dual-System GNSS Receiver[J], Telemetry and Remote Control Vol.2,2012. 